\title{
Electrical Impedance Tomography Based on a High-resolution Direction-of-arrival Estimation Algorithm
}

\author{
Jun Wen, Guoen Wei, and Xue Xiong \\ School of Computer, Electronics and Information, Guangxi University, \\ No. 100 Daxue East Road, Nanning 530004, China \\ (Received May 29, 2020; accepted August 12, 2020)
}

Keywords: EIT, DOA estimation, inverse problem, m-Capon algorithm, high resolution

The combination of artificial intelligence and medical imaging technology is profoundly affecting the development of medical imaging technology. Electrical impedance tomography (EIT) is a noninvasive imaging technology for estimating the internal impedance distribution of a body and is becoming a promising technology. In this paper, we focus on improving the EIT resolution by incorporating the direction-of-arrival (DOA) theory into the EIT model and use the DOA estimation algorithm to solve the inverse problem of EIT. The m-Capon algorithm proposed in this paper is based on a beamforming framework, there is no need to estimate the model order from the covariance matrix, and high resolution is obtained. The algorithms are simulated on the eidors platform of MATLAB. We use a resolution function, an image reconstruction quality function, and a correlation function as image evaluation functions. We compare the m-Capon algorithm with the back-projection (BP) algorithm, the Gauss-Newton (GN) algorithm, and a multiple signal classification (MUSIC)-like algorithm under different EIT models and signal-to-noise ratios (SNRs). Simulation results show that the EIT technology based on the DOA estimation algorithm used in this study is feasible, and the m-Capon imaging algorithm proposed in this paper gives higher resolution, stronger anti-jamming ability, and higher image quality than the other algorithms.

\section{Introduction}

In recent years, the combination of artificial intelligence and medical imaging technology has become a research hotspot of computer-aided diagnosis. Medical imaging systems with artificial intelligence will be an inevitable trend of medical imaging in the future. Electrical impedance tomography (EIT) is a newly developed and nondestructive imaging technology, ${ }^{(1,2)}$ which is promising for obtaining medical data in medical imaging systems. By applying a harmless and alternating current to an electrode array placed on the body surface, the resulting potential from boundary electrodes is obtained. On the basis of potential data, real-time object impedance distribution images are obtained through the reconstruction algorithm.

The EIT technology usually needs to solve forward and inverse problems. The solution of a forward problem, which is the variation of the potential distribution in the target domain

*Corresponding author: e-mail: wayen1994@foxmail.com

https://doi.org/10.18494/SAM.2020.2952 
from the known electrical impedance distribution data and injection currents, is unique. In fact, a forward problem in EIT is equivalent to solving the boundary value problem in an electromagnetic field, which can be defined by partial differential equations and boundary conditions. In EIT, the inverse problem involves using surface measurements of voltage, current data, and boundary excitation signals to solve the impedance distribution in an organism. This process, known as impedance image reconstruction, is a key part of EIT technology. EIT is widely used in the study of functional physiology and disease diagnosis because of its advantages of non-injury, functional imaging, and medical image monitoring. One of the main challenges is that the EIT reconstruction process is a typical nonlinear and ill-posed inverse problem. Traditional reconstruction algorithms are the back-projection (BP) algorithm, ${ }^{(3)}$ Gauss-Newton (GN) algorithm, ${ }^{(4)}$ modified Newton-Raphson (MNR) algorithm, ${ }^{(5)}$ Tikhonov regularization (TV) algorithm, ${ }^{(6)}$ and so on.

The direction-of-arrival (DOA) estimation algorithm is widely used in locating the signal source. For example, the multiple signal classification (MUSIC) algorithm is a DOA estimation algorithm with high resolution that is based on subspace decomposition, but it is necessary to know the number of signal sources in advance. To avoid this constraint and maintain high resolution, Zhang proposed a MUSIC-like algorithm, ${ }^{(7)}$ in which the number of sources need not be known, and a high-precision DOA estimation algorithm that can avoid performance deterioration due to the inaccurate estimation of the number of sources was developed. Borijindargoon introduced a mathematical theory for spatial spectrum estimation into the EIT model, then applied a MUSIC-like algorithm to solve the EIT inverse problem. ${ }^{(8)}$ Although a MUSIC-like algorithm has a clear positioning and imaging result for a target, there are more artifacts of other signals in the imaging domain, especially when the signal-to-noise ratio (SNR) is low.

In this paper, under the framework of the model proposed by Borijindargoon, the m-Capon algorithm is used for EIT, which is accurate in locating areas with changes in electrical impedance and in obtaining a high-quality reconstruction image.

\section{Methodology}

We consider the framework of the model proposed by Borijindargoon. ${ }^{(8)}$ Figure 1 shows a 16-electrode EIT system based on the method of adjacent current injection and voltage measurement.

For the forward problem, we use the complete electrode model (CEM) to map the relationship between the given impedance distribution and voltage measurements at the domain boundary. As shown in Fig. 1, the boundary voltage is measured using an array of electrodes.

A low-frequency alternating current with a typical amplitude of $1 \mathrm{~mA}$ is injected into a pair of electrodes $\varepsilon_{k}$ and $\varepsilon_{k+1}$ from different positions, and the electrical potential $U_{k}$ of $\varepsilon_{k}$ can be obtained from the Laplace equation. According to the method of adjacent current injection and voltage measurement, we obtain the $j$ th injection current from the $k$ th voltage $v^{j, k}$, where $v^{j, k}=U_{k}-U_{k+1}$ is the differential voltage between adjacent electrodes.

The CEM mathematical model is defined as Eqs. (1)-(5). This model includes an elliptic partial differential equation and some mixed boundary conditions. ${ }^{(9)}$ 


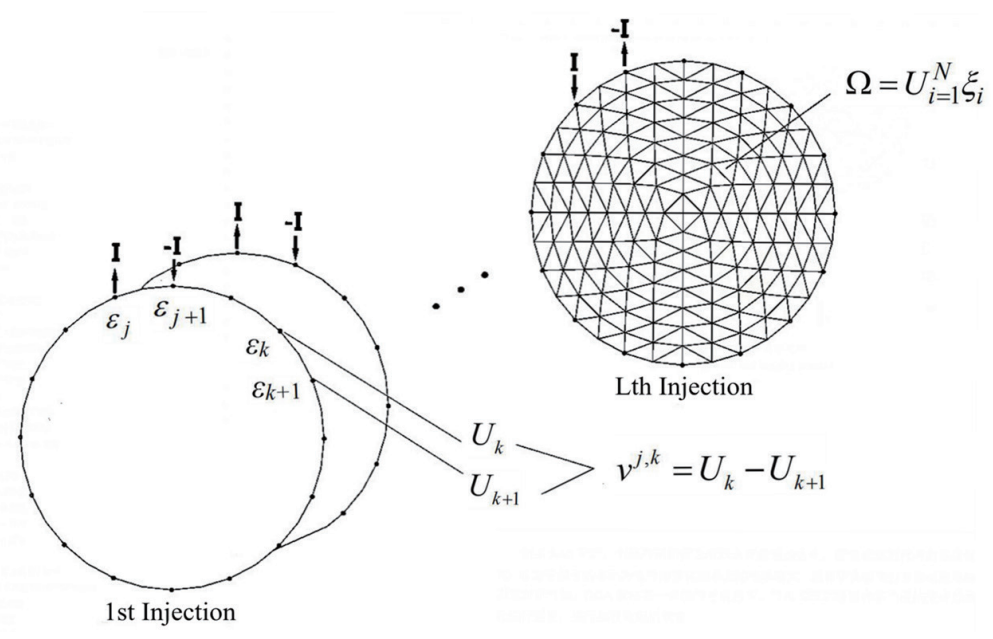

Fig. 1. Sixteen-electrode voltage measurement model for EIT electrodes.

$$
\begin{aligned}
& \nabla \cdot(\sigma \nabla \varphi)=0 \\
& \left(\varphi+z_{k} \sigma \frac{\partial \varphi}{\partial n}\right) \mid \varepsilon=U_{k}, k=1, \ldots, L \\
& \sigma \frac{\partial \varphi}{\partial n}=0 \\
& \int_{\varepsilon_{k}} \sigma \frac{\partial \varphi}{\partial n} d s=0, k \in\{1, \ldots, L\} \backslash\{j, j+1\} \\
& \int_{\mathcal{E}_{j}} \sigma \frac{\partial \phi}{\partial n} d s=I=-\int_{\mathcal{E}_{j+1}} \sigma \frac{\partial \phi}{\partial n} d s
\end{aligned}
$$

Here, $\varphi$ is the electrical potential distribution, $\sigma$ is the inner impedance distribution, $L$ represents the number of electrodes, $I$ is the injection current, $z_{k}$ is the contact impedance of electrode $\varepsilon_{k}$, and $\varepsilon_{j}$ and $\varepsilon_{j+1}$ represent a pair of adjacent current injection electrodes at the $j$ th current injection. In general, it is difficult to find an analytic solution to the forward problem. The numerical technique of the finite element method is required to solve Laplace's equation [Eq. (1)], where the circular domain $\Omega$ is divided into $N$ pixels $\left(\xi_{i}\right)$, that is, $\Omega=U_{i=1}^{N} \xi_{i}$.

The measurement vector $v$ can be expressed as

$$
v=\left[v^{1,1}, \ldots, v^{1, L}, \ldots, v^{L, 1}, \ldots, v^{L, L}\right]
$$


The length of boundary voltage data is $M=L(L-3)$. That is, the data length of a 16-electrode EIT system is 208.

For simplicity, the background impedance is defined as $\sigma=1$, when the impedance changes as $\sigma \rightarrow \sigma+\Delta \sigma$ and the electrical potential changes as $\varphi \rightarrow \varphi+\Delta \varphi$. Equation (1) can be rewritten as ${ }^{(10)}$

$$
\begin{aligned}
& \nabla \cdot[(\sigma+\Delta \sigma) \nabla(\varphi+\Delta \varphi)]=0, \\
& \nabla \cdot \nabla(\Delta \varphi)=-\nabla \cdot\left(\left.\Delta \sigma\right|_{p} \nabla \varphi\right) .
\end{aligned}
$$

The change in the impedance of the $P$ th pixel in the domain produces a disturbance potential $\Delta \phi$. A snapshot of the signal received at the boundary can be modeled linearly as ${ }^{(8)}$

$$
x(n)=A(\Xi) s(n)+n(n),
$$

where $n=t_{k}, x(n) \in R^{M \times 1}$ is a snapshot of the voltage vector, $A(\Xi) \in R^{M \times N}$ is a sensing matrix (Jacobian matrix), and $s(n) \in R^{N \times 1}$ and $n(n) \in R^{M \times 1}$ denote the impedance vector (source vector) and noise vector, respectively. Figure 2 shows snapshots of two circular signal source targets in a field $\Omega$ at different times $t_{k}$.

The changes in impedance are presented as different grayscale colors, and every snapshot of a domain image is different when the signal source impedance changes. Thus, the element values in the source matrix $S \in R^{N \times k}$ are presented as different gray values, as shown in Eq. (10) and Fig. 3.

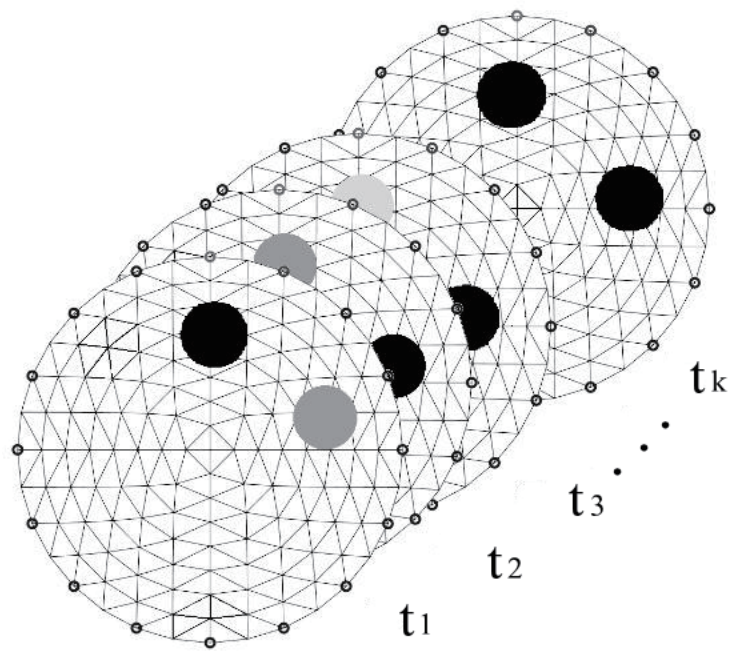

Fig. 2. Snapshots of two signal source targets at different times.

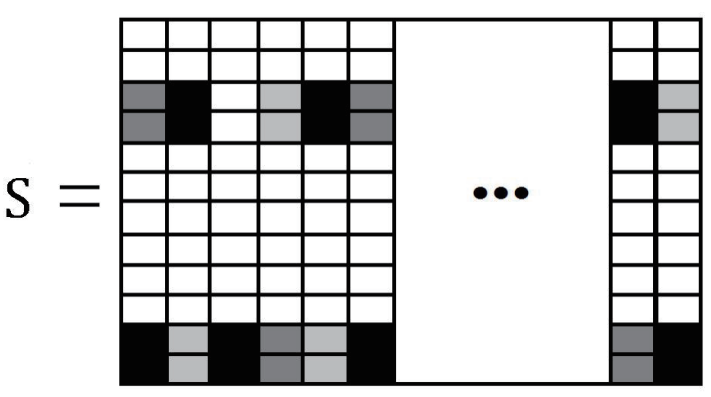

Fig. 3. Grayscale representation of impedance variation inside the domain over different snapshots. 


$$
S=\left[\begin{array}{c}
s\left(t_{1}\right) \\
s\left(t_{2}\right) \\
\vdots \\
s\left(t_{k}\right)
\end{array}\right]^{T}=\left[\begin{array}{ccccc}
\Delta \sigma\left(\xi_{1}, t_{1}\right) & \Delta \sigma\left(\xi_{1}, t_{2}\right) & \Delta \sigma\left(\xi_{1}, t_{3}\right) & \cdots & \Delta \sigma\left(\xi_{1}, t_{k}\right) \\
\Delta \sigma\left(\xi_{2}, t_{1}\right) & \Delta \sigma\left(\xi_{2}, t_{2}\right) & \Delta \sigma\left(\xi_{2}, t_{3}\right) & \cdots & \Delta \sigma\left(\xi_{2}, t_{k}\right) \\
\vdots & \vdots & \vdots & \ddots & \vdots \\
\Delta \sigma\left(\xi_{N}, t_{1}\right) & \Delta \sigma\left(\xi_{N}, t_{2}\right) & \Delta \sigma\left(\xi_{N}, t_{3}\right) & \cdots & \Delta \sigma\left(\xi_{N}, t_{k}\right)
\end{array}\right]
$$

Therefore, the model of the snapshots of signals received at the boundary can also be expressed as ${ }^{(8)}$

$$
\begin{gathered}
X=A(\Xi) S+N_{n} \rightarrow X=A_{B}(\Xi) S_{B}+N_{n}, \\
A(\Xi)=A_{B}(\Xi)=\left[a\left(\xi_{1}\right), a\left(\xi_{2}\right), \ldots, a\left(\xi_{N}\right)\right], \\
a\left(\xi_{i}\right)=\left[g_{1}^{1}\left(\xi_{i}\right), \ldots, g_{L-3}^{1}\left(\xi_{i}\right), \ldots, g_{1}^{L}\left(\xi_{i}\right), \ldots, g_{L-3}^{L}\left(\xi_{i}\right)\right]^{H}, \\
g_{k}^{j}=\frac{\int_{\xi_{i}} \nabla \varphi^{k} \nabla \varphi^{j} d r}{\sqrt{\sum_{j=1}^{L} \sum_{k \notin j-1, j, j+1}\left|\int_{\xi_{i}} \nabla \varphi^{k} \nabla \varphi^{j} d r\right|}},
\end{gathered}
$$

where $X=\left[\begin{array}{llll}x\left(t_{1}\right) & x\left(t_{2}\right) & \cdots & x\left(t_{k}\right)\end{array}\right]_{M \times k}$ is defined as the voltage matrix, $N_{n} \in R^{M \times k}$ denotes the sum of the additive white Gaussian noise (AWGN) and the unmodelled error matrix, $A(\Xi) \in R^{M \times N}$ is a manifold matrix, $B$ represents the nonzero row index set of the source matrix $S, a\left(\xi_{i}\right) \in R^{M \times 1}$ is the steering vector, and $g_{k}^{j}\left(\xi_{i}\right)$ is the expected normalized potential change caused by the $k$ th induced polar source.

It is assumed that when the number of snapshots approaches infinity and the mean of the data matrix is zero, the covariance matrix of the snapshot data is

$$
R=A_{B}(\Xi) R_{S} A_{B}(\Xi)^{H}+\sigma_{n}^{2} I_{M}
$$

where $R=E\left(X X^{H}\right), R_{S}=E\left(S_{B} S_{B}^{H}\right)$ is the signal covariance matrix, and $\sigma_{n}^{2} I_{M}=E\left(N N^{H}\right)$ is the noise covariance matrix.

\section{3. m-Capon Algorithm}

In this section, the m-Capon algorithm for tackling the EIT inverse problem is devised. The m-Capon algorithm is a super-resolution DOA estimation algorithm based on the Capon algorithm. ${ }^{(11,12)}$ The advantages of the m-Capon algorithm are threefold: (a) There is no need to estimate the number of source signals. (b) There is no need to carry out signal feature decomposition. (c) It maintains a high resolution in a time-varying environment when the samples are small. 


\subsection{Signal model of Capon algorithm}

The Capon beamformer, known as the minimum variance distortionless response (MVDR) beamformer, uses the degree of freedom (DOF) to minimize the output power of the interference signal, thus eliminating the influence of the interference signal and maintaining a constant gain in the observation direction.

In radar array signal processing, the received signal in the antenna array is expressed as ${ }^{(13)}$

$$
X(t)=A S(t)+N(t),
$$

where $X(t) \in R^{M \times 1}$ is the vector of a snapshot array data, $A \in R^{M \times K}$ is the manifold matrix, $S(t) \in R^{K \times 1}$ is the vector of the signal, $N(t) \in R^{M \times 1}$ is the noise, $M$ represents the number of antennas, and $K$ represents the number of source signals. The optimization problem of the Capon beamformer is expressed as

$$
\min _{w} w^{H} R w,
$$

where the constraint is

$$
w^{H} a(\theta)=1 .
$$

Here, covariance matrix $R=E\left(X X^{H}\right), w$ is the optimal weighted vector, and $\theta$ is the signal incident angle. The Capon beamformer can keep the power of the desired signal unchanged while minimizing the power contributed by noise and interference.

The optimal weighted vector $w$ of Eqs. (17) and (18) is solved by the Lagrange multiplier method,

$$
L(w, \lambda)=w^{H} R w+\lambda\left(w^{H} a(\theta)-1\right),
$$

where $\lambda$ is the Lagrange multiplier and $w$ is optimized to minimize $L(w, \lambda)$. Hence, $w$ can be expressed as

$$
w_{C a p}=\frac{R^{-1} a(\theta)}{a(\theta) R^{-1} a(\theta)} .
$$

The spatial spectrum of the Capon beamformer is expressed as

$$
P_{\text {Cap }}=w_{\text {Cap }}{ }^{H} R w_{\text {Cap }}=\frac{1}{a^{H}(\theta) R^{-1} a(\theta)},
$$

where $R=E\left(X X^{T}\right)$ is the covariance matrix and $a(\theta)$ is the steering vector. 


\subsection{Signal model of $\mathrm{m}$-Capon algorithm}

The Capon algorithm does not need to predict the number of sources, but it is limited by the Rayleigh limit and has poor angular resolution. The MUSIC algorithm has super-resolution performance, but it needs to estimate the number of sources and decompose the covariance matrix eigenvalues. Therefore, we apply the m-Capon algorithm, which is obtained by combining the advantages of the Capon and MUSIC algorithms to the reconstruction process.

The array covariance matrix $R$ can be expressed as ${ }^{(14)}$

$$
\begin{aligned}
R & =\left[\begin{array}{ll}
U_{S} & U_{n}
\end{array}\right]\left[\begin{array}{cc}
\Lambda & 0 \\
0 & \sigma_{n}^{2} I
\end{array}\right]\left[\begin{array}{c}
U_{S}^{H} \\
U_{n}^{H}
\end{array}\right] \\
& =U_{S} \Lambda_{S} U_{S}^{H}+\sigma_{n}^{2} U_{n} U_{n}^{H},
\end{aligned}
$$

where $U_{s}$ is a signal subspace, $U_{n}$ is a noise subspace, and $\Lambda_{\mathrm{s}}$ is a diagonal matrix composed of large eigenvalues of the covariance matrix $R$. This equation can be expressed as

$$
\sigma_{n}^{2 m} R^{-m}=U_{n} U_{n}^{H}+U_{S} \operatorname{diag}\left\{\left(\frac{\sigma_{n}^{2}}{\lambda_{i}}\right)^{m}\right\}^{2} U_{S}^{H}
$$

where $m$ is an integer and $\left(\frac{\sigma_{n}^{2}}{\lambda_{i}^{2}}\right)<1$. When $m$ approaches infinity, Eq. (23) can be expressed as

$$
\lim _{m \rightarrow \infty} \sigma_{n}^{2 m} R^{-m}=U_{n} U_{n}^{H}
$$

Therefore, the optimization equation for the DOA estimation of the m-Capon algorithm is ${ }^{(8)}$

$$
\begin{aligned}
\theta & =\arg \max _{\theta}\left\{\prod_{i=1}^{m} \frac{1}{a^{H}(\theta) R^{-1} a(\theta)}\right\} \\
& =\arg \max _{\theta}\left\{\frac{1}{a^{H}(\theta) R^{-m} a(\theta)}\right\} \\
& =\underset{\theta}{\arg \min _{\theta}}\left\{a^{H}(\theta) R^{-m} a(\theta)\right\} .
\end{aligned}
$$

By introducing the m-Capon algorithm into the EIT model, the spatial spectrum of the m-Capon algorithm can be expressed as 


$$
\begin{aligned}
P_{\mathrm{m}-C \mathrm{ap}} & =\arg \max _{\xi}\left\{\prod_{i=1}^{m} \frac{1}{a^{H}(\xi) R^{-1} a(\xi)}\right\} \\
& =\arg \max _{\xi}\left\{\frac{1}{a^{H}(\xi) R^{-m} a(\xi)}\right\} \\
& =\arg \min _{\xi}\left\{a^{H}(\xi) R^{-m} a(\xi)\right\} .
\end{aligned}
$$

DOA technology can find the locations of the pixels with changing impedance, $\xi_{i} \in \Omega(i=1,2, \ldots, N)$, without needing to predict the number of pixels with changing electrical impedance. In the diagram of peak search, the numbered cell corresponding to the spectral peak can be regarded as the cell whose resistance has changed.

\section{Simulation}

In this section, we present the reconstruction images and evaluate the performance of the proposed method and classical algorithms by various simulations.

\subsection{Imaging with m-Capon algorithm}

A simulation experiment was carried out with the EIT imaging open-source software platform eidors3.9.1 on MATLAB. ${ }^{(15)}$ As shown in Fig. 4, a 16-electrode two-dimensional circular domain model with an 8-layer partition and a finite element partition with 256 pixels was used. Therefore, the voltage measurement matrix $X^{208 \times 2}$, the covariance matrix $R^{208 \times 208}$, and the Jacobian matrix $J^{208 \times 256}$ (array manifold) can be obtained. We assume that the injection current $I$ is $1 \mathrm{~mA}$, the SNR is $10 \mathrm{~dB}$, and the background impedance is $\sigma_{1}=1$. The EIT finite element model is shown in Fig. 4, the electrical impedances of the target pixels are numbered 60,100 , and 200, and the simulated target impedance is $\sigma_{2}=100$.

In this paper, the m-Capon algorithm reconstructs the impedance image from boundary voltage measurements. The results of the peak search are shown in Fig. 5, where the abscissa corresponds to the number of pixel blocks in the circular domain and the ordinate corresponds to the spatial spectral intensity. From the spectral search graph, the spatial spectral intensity of the pixel blocks numbered 60,100 , and 200 is the highest. It can be considered as the number of target pixel blocks.

From the different spatial spectral intensities of the different numbered pixel blocks, the reconstructed image can be obtained by assigning the corresponding numbered pixel blocks. As shown in Fig. 6, the m-Capon algorithm can locate and image multiple areas (target areas) with varying impedance. 


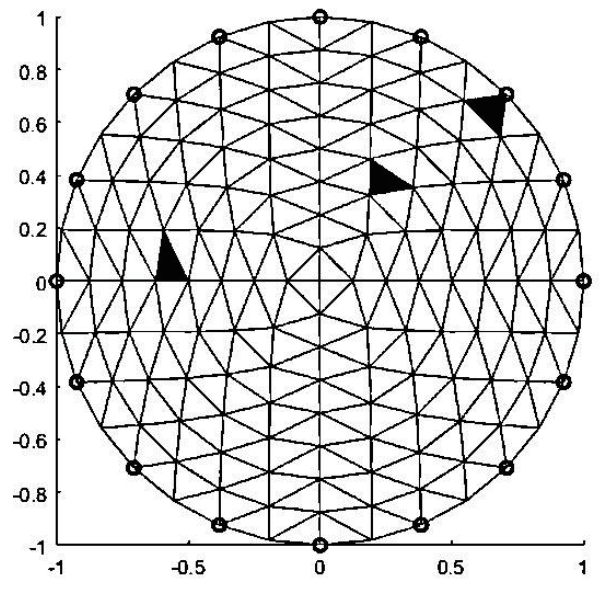

Fig. 4. Target model.

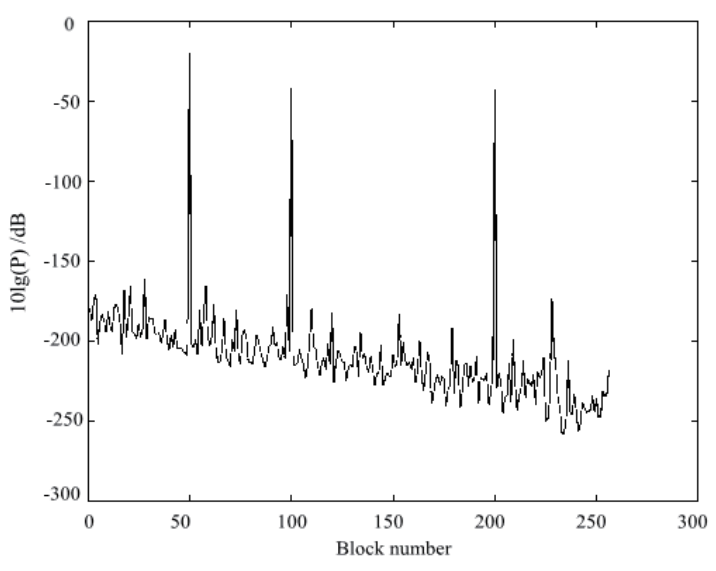

Fig. 5. Results of peak search.

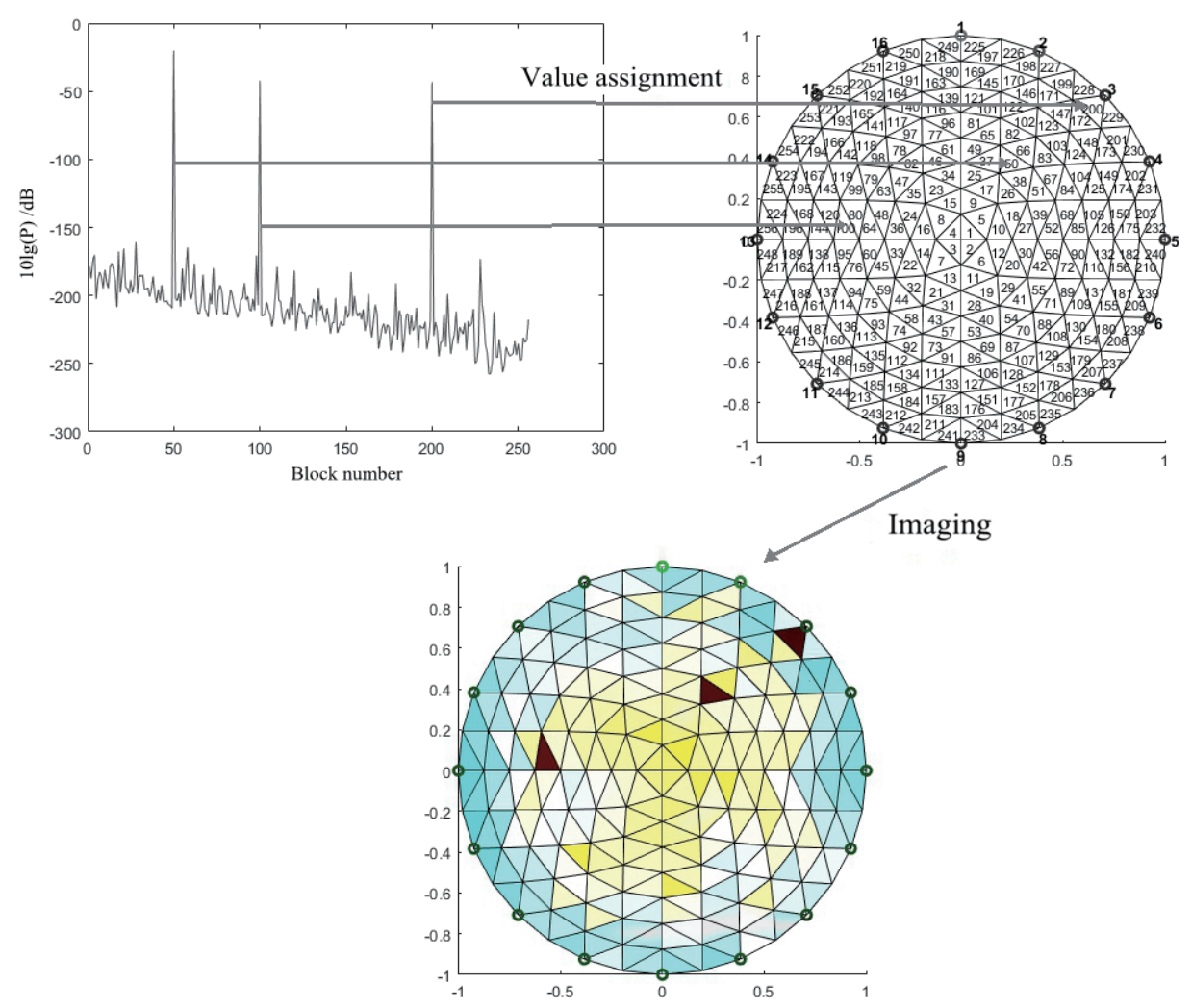

Fig. 6. (Color online) Assignment and imaging processes.

\subsection{Comparisons and evaluation of algorithms}

\subsubsection{Functions for evaluating image quality}

To describe the performance of the proposed reconstruction method, we introduced three metrics to access the image quality. These metrics well reflect the difference in image information among different imaging algorithms. 
a. Resolution function $(R E S)^{(16)}$

$$
R E S=\frac{n_{Y}}{N}
$$

Here, $N$ is the total number of pixels, $n_{Y}$ is the number of target pixels after the threshold processing of the reconstructed image, and RES is proportional to the resolution of the system.

b. Image reconstruction quality function $(D)^{(17)}$

$$
D=\frac{\sum_{p=1}^{N}\left|G_{y}(p)-G_{x}(p)\right|}{N}, P=1,2, \ldots, N
$$

The total number of pixel blocks is $N, G_{x}(p)$ is the normalized impedance of each pixel block in the original image model $D$, and $G_{y}(p)$ is the normalized impedance of each pixel block in the reconstructed image. The smaller the value of $D$, the smaller the difference between the image and the model and the higher the quality of image reconstruction.

c. Correlation function $(C O R R)$

$$
\operatorname{CORR}(X, Y)=\left|\frac{\sum_{n=1}^{N}\left(X_{n}-\sigma_{X}\right)\left(Y_{n}-\sigma_{Y}\right)}{\sqrt{\left(\sum_{n=1}^{N}\left(X_{n}-\sigma_{X}\right)^{2}\right)\left(\sum_{n=1}^{N}\left(Y_{n}-\sigma_{Y}\right)^{2}\right)}}\right|
$$

Here, $N$ is the total number of elements, $X$ is the original image, $Y$ is the reconstructed image, and $\sigma X$ and $\sigma Y$ are the mean values of the impedance in images $X$ and $Y$, respectively. When CORR is closer to 0 , the similarity between the original image and the reconstructed image is lower and the degree of reconstruction is also lower, and when the CORR value is closer to 1 , the similarity between the original image and the reconstructed image is higher, indicating a higher degree of reconstruction.

\subsubsection{Simulation and evaluation of different imaging algorithms}

To evaluate the performance of the proposed method compared with other algorithms, four original models, Models 1-4, are constructed. The number of subdivision pixels in each model is 256. When the impedance is unchanged, we set the impedance of domain as $\sigma_{1}=1$ and that of the target domain as $\sigma_{2}=100$. Four algorithms, BP, GN, MUSIC-like, and m-Capon, are 
used to reconstruct and image the models when SNRs are $-10,0,20$, and $50 \mathrm{~dB}$, the results of which are shown in Fig. 7.

We present reconstruction images illustrating the performance of the m-Capon algorithm compared with the BP, GN, and MUSIC-like algorithms. Note that the performance characteristics of the BP, GN, and MUSIC-like algorithms deteriorate and the number of image artifacts increases when the target moves from the edge to the center even at a high SNR. However, the m-Capon algorithm is able to reconstruct a clear target and shows the best performance.

In addition, the performance of each algorithm is measured using image evaluation functions of RES, D, and CORR. The evaluation results for the above four algorithms under different target numbers with the image evaluation functions are shown in the figures below.

From Fig. 7, we can see the reconstructed images of Models 1-3 for a single target. The BP and GN algorithms can estimate the position of the imaging target but cannot judge its shape even in the case of a high SNR. Both the MUSIC-like and m-Capon algorithms can identify the position and the imaging target shape at low and high SNRs. However, the imaging

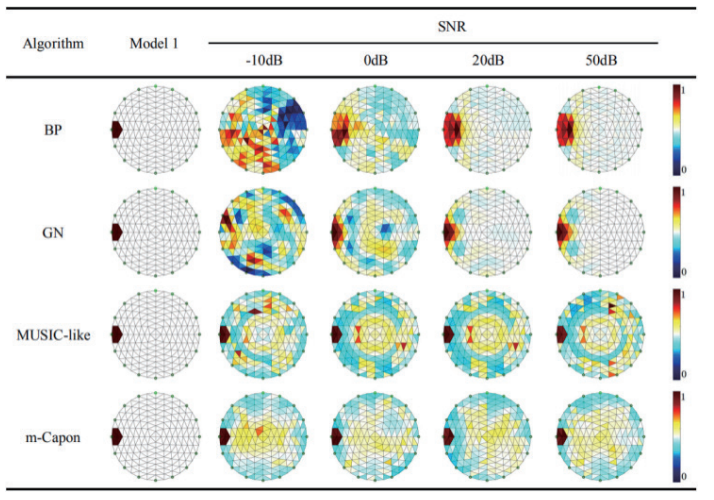

(a)

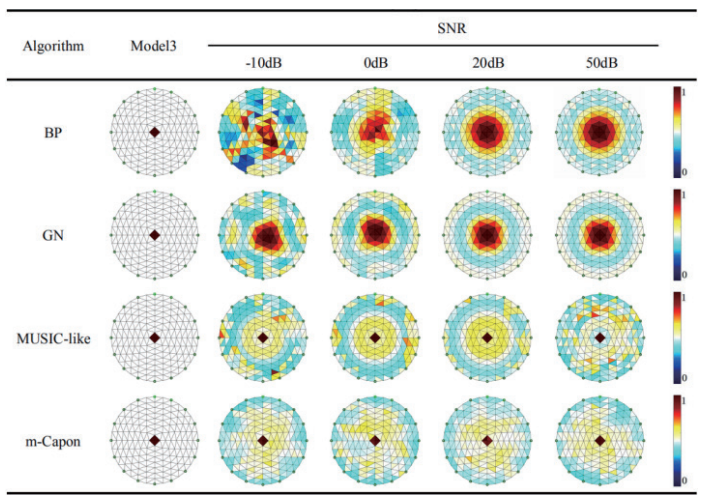

(c)

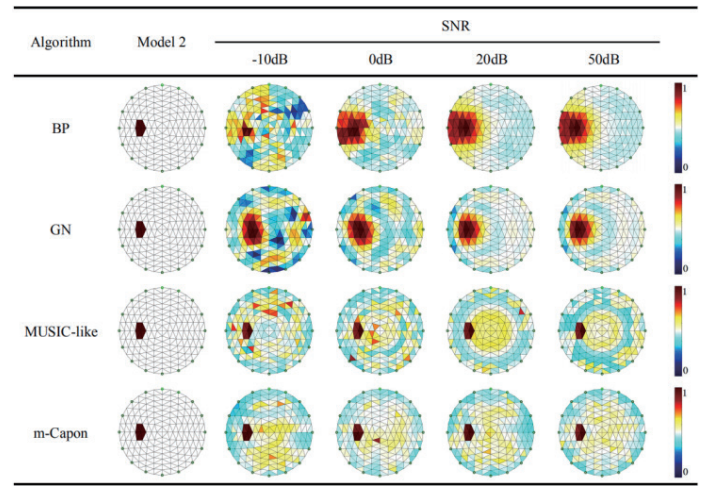

(b)

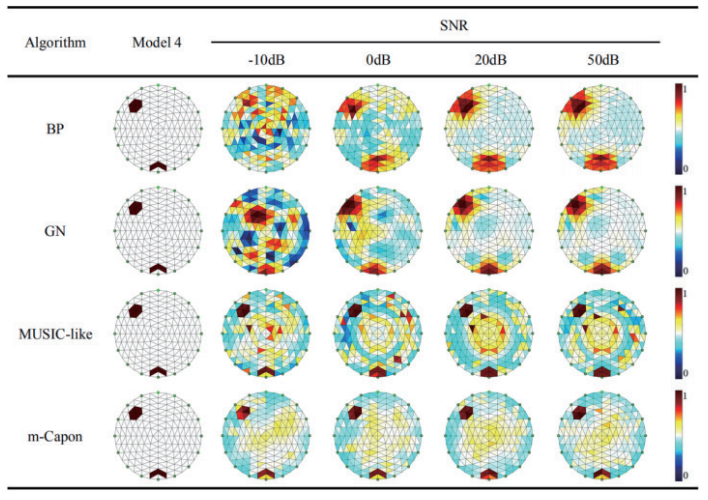

(d)

Fig. 7. (Color online) Comparison of imaging models obtained with various algorithms: (a) Model 1, (b) Model 2, (c) Model 3, and (d) Model 4. 
performance of the m-Capon algorithm is higher than that of the MUSIC-like algorithm; furthermore, the former can reconstruct a clear target and reduce the number of artifacts in the area outside the target.

Model 4 is a two-target model, and it can be seen from its recomposition that the reconstruction results of the BP and GN algorithms on the double target are basically consistent with those on the single target. The MUSIC-like algorithm can identify the position and target shape, but there is more interference in the area outside the target. In contrast, the m-Capon algorithm again has the highest imaging quality.

Figures $8-11$ show the reconstructed evaluation curves for each model, revealing the reconstruction performance of each algorithm. With increasing SNR, the value of each evaluation function tends to become stable. For the resolution RES comparison curve, the resolutions of the BP and GN algorithms decrease with increasing SNR and eventually become stable. Although the curve of the MUSIC-like algorithm is close to that of the m-Capon algorithm, the resolution of the different models for the $\mathrm{m}$-Capon algorithm is higher than that of the MUSIC-like algorithm under different SNRs. For the contrast curve of image quality $D$, when the SNR is small, the image quality of the BP and GN algorithms is poor. With increasing SNR, the image quality improves and tends to become stable, while the image quality of the MUSIC-like and m-Capon algorithms is relatively stable regardless of the SNR. For the CORR comparison curve of image similarity, the GN algorithm has a high CORR value equivalent to that of the $\mathrm{m}$-Capon algorithm when the target is near the boundary and the SNR is high. However, when the target is far from the boundary, the image quality is poor and the CORR value is lower than that of the m-Capon algorithm. The CORR value of the BP and MUSIC-like algorithms increases and tends to become stable with increasing SNR, but it is lower than that of the m-Capon algorithm. In conclusion, the image CORR value of the $\mathrm{m}$-Capon algorithm remains the best regardless of the SNR and whether the target is near to or far from the boundary, indicating that the reconstruction obtained by the m-Capon algorithm is the most similar to the original model.

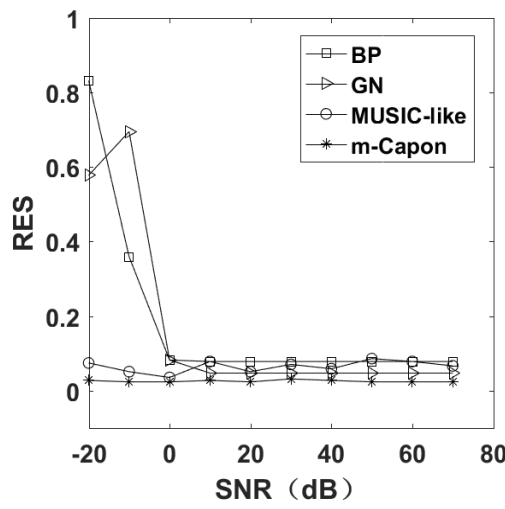

(a)

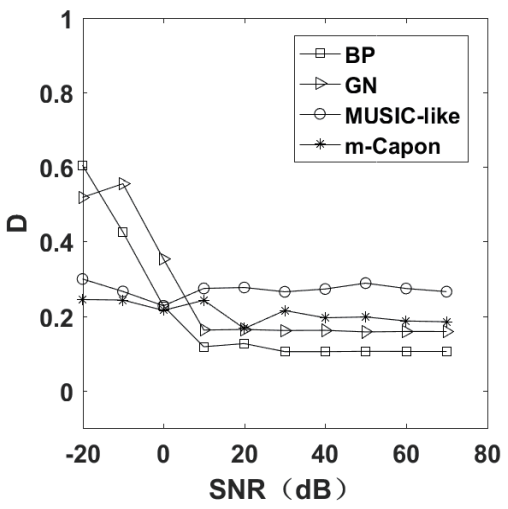

(b)

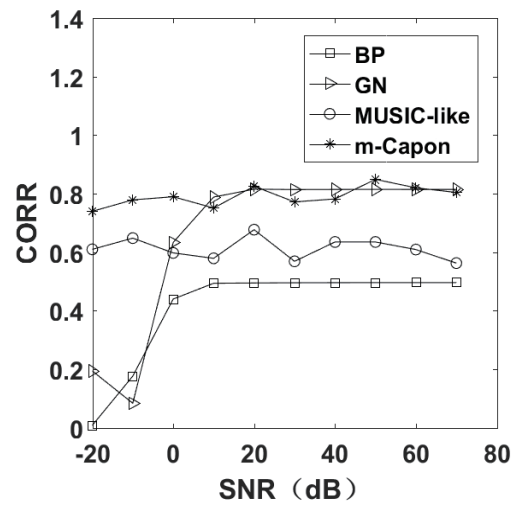

(c)

Fig. 8. Reconstruction image evaluation of Model 1: (a) result of resolution function $R E S$, (b) result of image reconstruction quality function $D$, and (c) result of correlation function CORR. $\mathrm{SNR}=[-20,-10,0,10,20,30,40,50,60,70]$. 


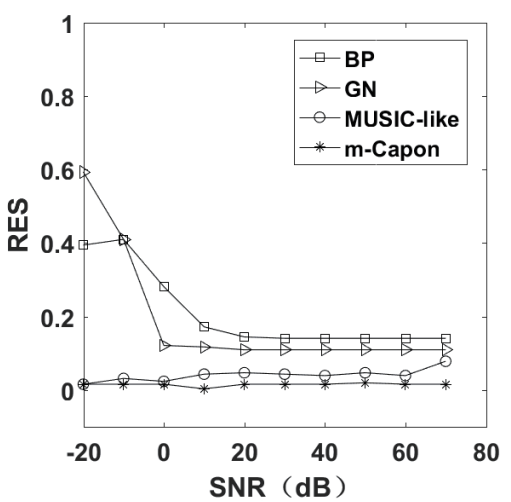

(a)

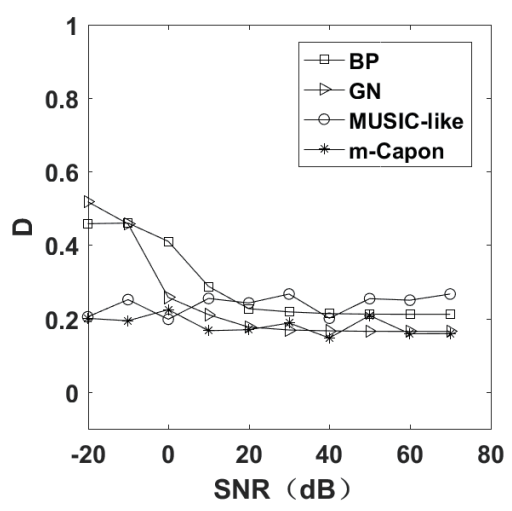

(b)

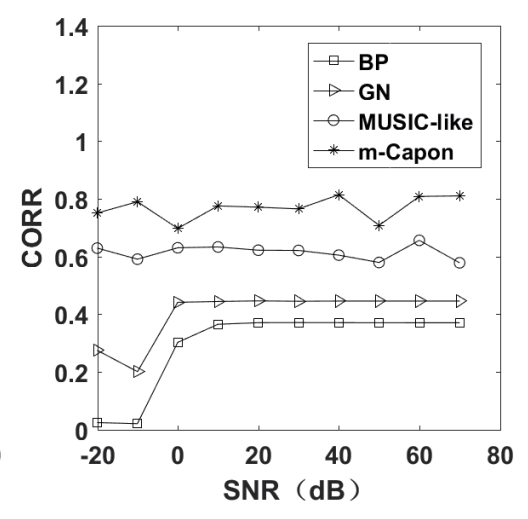

(c)

Fig. 9. Reconstruction image evaluation of Model 2: (a) result of resolution function $R E S$, (b) result of image reconstruction quality function $D$, and (c) result of correlation function CORR. SNR $=[-20,-10,0,10,20,30,40,50,60,70]$.

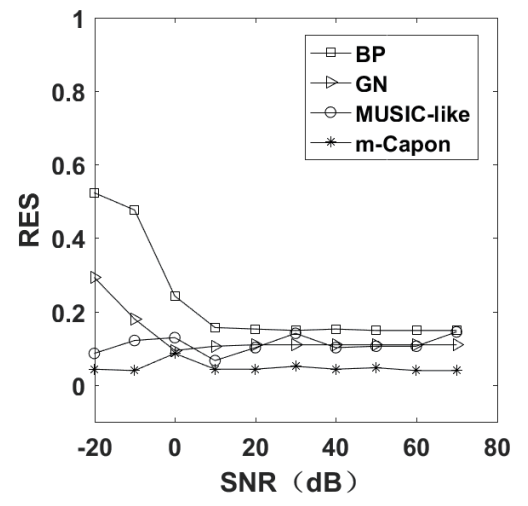

(a)

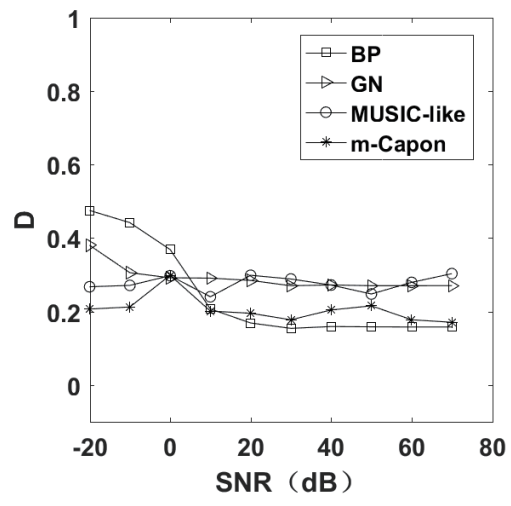

(b)

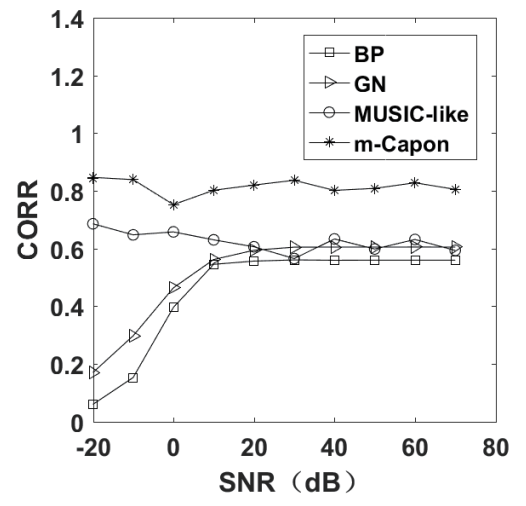

(c)

Fig. 10. Reconstruction image evaluation of Model 3: (a) result of resolution function $R E S$, (b) result of image reconstruction quality function $D$, and (c) result of correlation function CORR. $\mathrm{SNR}=[-20,-10,0,10,20,30,40,50,60,70]$.

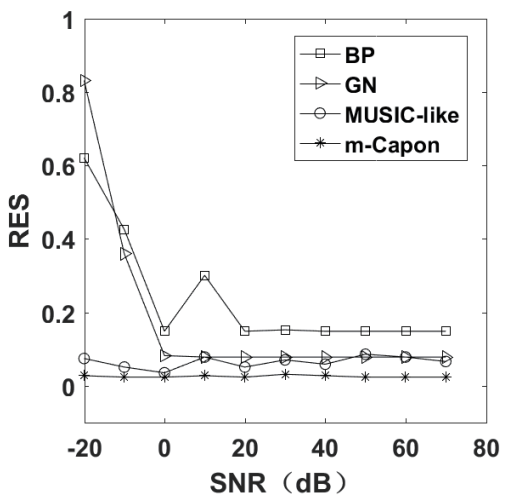

(a)

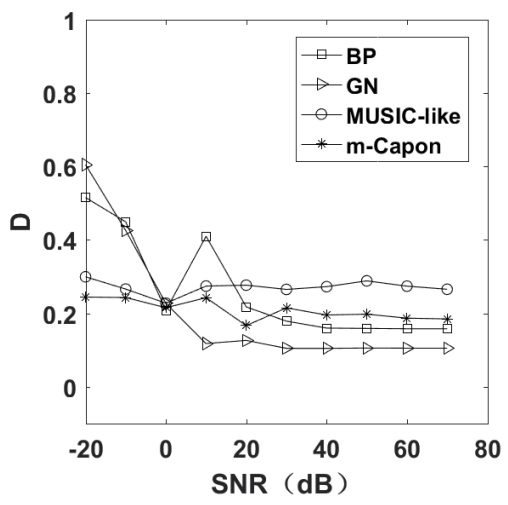

(b)

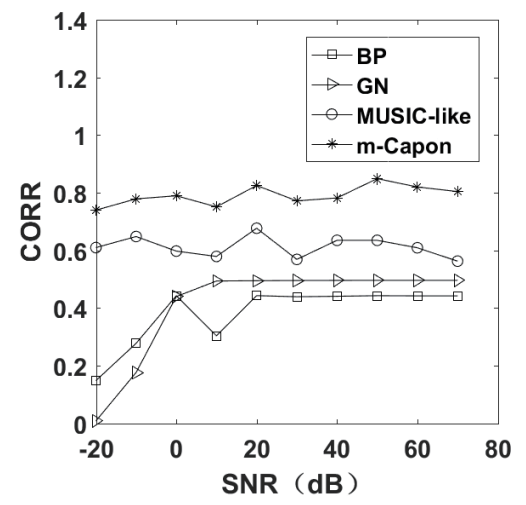

(c)

Fig. 11. Reconstruction image evaluation of Model 4: (a) result of resolution function $R E S$, (b) result of image reconstruction quality function $D$, and (c) result of correlation function CORR. $\mathrm{SNR}=[-20,-10,0,10,20,30,40,50,60,70]$. 
Compared with the other three algorithms, the m-Capon algorithm has higher resolution, stronger anti-interference ability, and higher image quality and similarity to the imaging result for single and multiple target areas with impedance variation.

\section{Conclusions}

In this paper, the DOA estimation theory is introduced into the EIT technology, and the $\mathrm{m}$-Capon algorithm is used to locate areas with the changing impedance on the eidors platform. Compared with the GN, BP, and MUSIC-like algorithms, the m-Capon algorithm used in this study has smaller resolution and image reconstruction quality function values and a larger correlation function value in many cases. This means that the performance of the m-Capon algorithm is more stable than those of the other algorithms, that is, the m-Capon algorithm can locate areas with changing impedance more accurately and with higher imaging quality.

\section{Acknowledgments}

The study was supported by the National Natural Science Foundation of China (General Program Projects Grant No. 61871138), the Young Scientists Fund (Grant No. 41806211), and the GuangXi Key R\&D Program (Grant No. GuiKe AB18126094).

\section{References}

1 H. Jain, D. Isaacson, P. M. Edic, and J. C. Newell: IEEE. Trans. Biomed. Eng. 44 (1997) 1051. https://doi. org $/ 10.1109 / 10.641332$

2 J. C. de Munck, T. J. C. Faes, and R. M. Heethaar: IEEE. Trans. Biomed. Eng. 47 (2000) 792. https://doi. org $/ 10.1109 / 10.844230$

3 X. F. Liu, Q. W. Li, H. C. Lin, P. F. Wu, and M. D. Wang: Sens. Mater. 31 (2019) 4013. https://doi.org/10.18494/ SAM.2019.2515

4 B. Bernhard, H. Karl, and H. Helmut: Physiol. Meas. 24 (2003) 437. https://doi.org/10.1088/0967-3334/24/2/355

5 C. Y. Kim, J. M. Kang, J. H. Kim, B. Y. Choi, and K. Y. Kim: J. Korean Phys. Soc. 61 (2012) 1199. https://doi. org/10.3938/ jkps.61.1199

6 Y. Peng, Y. L. Mo: J. Biomed. Eng. 20 (2003) 571. https://doi.org/110.3321/j.issn:1001-5515.2003.04.001

7 Y. Zhang and B. P. Ng: IEEE Trans. Signal Process. 58 (2010) 1668. https://doi.org/ 10.1109/TSP.2009.2037074

8 B. Narong, N. B. Poh, and R. Susanto: IEEE Trans. Ind. Electron. 66 (2019) 4661. https://doi.org/10.1109/ TIE.2018.2863196

9 D. Sarmany, M. A. Botchev, and J. J. W. van der Vegt: Comput. Math. Appl. 65 (2013) 528. https://doi. org/10.1016/j.camwa.2012.05.023

10 D. S. Holder: Electrical Impedance Tomography: Methods, History and Applications, D. S. Holder, Ed. (IOP Publishing, Bristol, U.K, 2004) 1st ed., Chap. 1. https://doi.org/10.1016/10.1118/1.1995712

11 J. Li, S. Petre, and Z. S. Wang: IEEE Trans. Signal Process. 52 (2005) 2407. https://doi.org/10.1109/ TSP.2004.831998

12 A. Hassanien, S. Shahbazpanahi, and A. B. Gershman: IEEE Trans. Signal Process. 52 (2004) 280. https://doi. org/10.1109/tsp.2003.820089

13 F. Li, H. Liu, and R. Vaccaro: IEEE Trans. Aerosp. Electron. Syst. 24 (1993) 1170. https://doi. org/10.1109/7.259520

14 T. L. Zhang, Y. Liu, and G. S. Liao: J. Electron. Inf. Technol. 30 (2008) 375. https://doi.org/10.3724/ SP.J.1146.2006.01020

15 A . Andy and L. William: Physiol. Meas. 27 (2006) S25. https://doi.org/10.1088/0967-3334/27/5/S03

16 A. Andy, A. John, and B. Richard: Physiol. Meas. 30 (2009) S35. https://doi.org/10.1088/0967-3334/30/6/S03

17 J. Deng, Y. Wang, and J. H. Lv: Chin. Med. Equip. J. 31 (2010) 1. https://doi.org/CNKI:SUN:YNWS.0.2010-05-005 


\section{About the Authors}

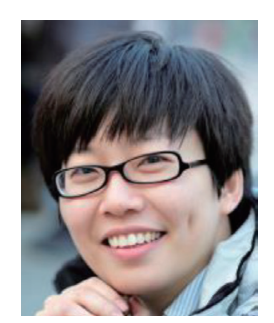

Jun Wen received her B.Sc. and Ph.D. degrees in electronics engineering from Xidian University, Xi'an, China, in 2006 and 2011, respectively. She was with the School of Electronic Engineering, Xidian University, from 2011 to 2013 as a lecturer. Since 2014, she has been with the School of Computer and Electrical Information, Nanning, China, as a professor. Her research interests include space-time adaptive processing and SAR ground moving target indication.

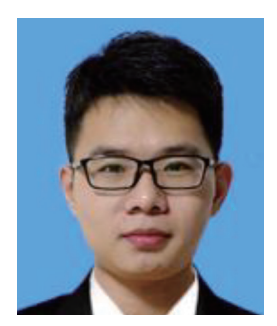

Guoen Wei received his B.S. degrees in electronic science and technology from Guilin University of Electronic Technology, Guilin, China, in 2016. He is currently pursuing an M.S. degree in electronics and communication engineering at Guangxi University, Nanning, China. His research interests include radar signal processing and electrical impedance tomography.

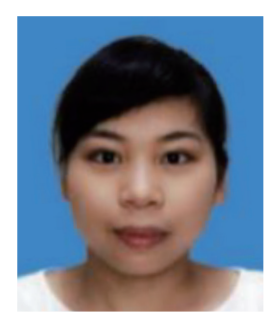

Xue Xiong received her B.S. degrees in electronics and information engineering from Guangxi University of Science and Technology, Liuzhou, China, in 2018. She is currently pursuing her M.S. degree in electronics and communication engineering from Guangxi University, Nanning, China. Her research interests include wireless communication, signal processing, and electrical impedance tomography. 
